Archives de sciences sociales des religions

176 | octobre-décembre 2016

Bulletin Bibliographique

\title{
Françoise Micheau, Les débuts de l'Islam. Jalons pour une nouvelle histoire
}

Paris, Téraèdre, 2012, 260 p.

Mohammed Hocine Benkheira

\section{CpenEdition}

\section{Journals}

Édition électronique

URL : http://journals.openedition.org/assr/28307

DOI : $10.4000 /$ assr.28307

ISSN : $1777-5825$

Éditeur

Éditions de l'EHESS

Édition imprimée

Date de publication : 31 décembre 2016

Pagination : 358

ISSN : 0335-5985

Référence électronique

Mohammed Hocine Benkheira, "Françoise Micheau, Les débuts de l'Islam. Jalons pour une nouvelle histoire », Archives de sciences sociales des religions [En ligne], 176 | octobre-décembre 2016, mis en ligne le 17 juillet 2017, consulté le 23 septembre 2020. URL : http://journals.openedition.org/assr/ 28307 ; DOI : https://doi.org/10.4000/assr.28307

Ce document a été généré automatiquement le 23 septembre 2020.

(C) Archives de sciences sociales des religions 


\title{
Françoise Micheau, Les débuts de l'Islam. Jalons pour une nouvelle histoire
}

Paris, Téraèdre, 2012, 260 p.

\author{
Mohammed Hocine Benkheira
}

\section{RÉFÉRENCE}

Françoise Micheau, Les débuts de l'Islam. Jalons pour une nouvelle histoire, Paris, Téraèdre, 2012, 260 p.

1 Voici un livre qui se propose de présenter des recherches récentes, notamment en langue anglaise, qui renouvellent la vision de l'islam primitif. Ces recherches sont souvent inaccessibles pour le lecteur francophone non spécialiste, car jamais traduites en français. Par exemple, les ouvrages de John Wansbrough ne sont toujours pas accessibles en français. Il en est de même des ouvrages de ses émules, comme le fameux Hagarism de Patricia Crone et Michael Cook. Il faut observer que les livres en question ne sont que la partie émergée de l'iceberg : c'est dans les revues que l'on peut prendre connaissance des recherches les plus novatrices. L'ouvrage comprend 8 chapitres. On n'évoquera pas le huitième chapitre, qui a trait à l'histoire de la Syrie et qui est éloigné du phénomène religieux.

2 Le premier chapitre aborde la question des origines de l'historiographie musulmane. L'auteur présente principalement les thèses de Fred Donner, Gregor Schoeler et Antoine Borrut. On peut regretter que certains auteurs importants ne soient même pas signalés: nous pensons notamment à Wadad al-Qadi. La thèse du premier est que l'écriture de l'histoire a servi «des fins de légitimation, dans un contexte de débats théologiques et de rivalités politiques » (p.31). Cet auteur a sans aucun doute raison, mais la réponse qu'il donne est bien sûr insuffisante, car l'écriture de l'histoire suppose une conscience de soi, c'est-à-dire la conscience de constituer " un seul être ", plus 
exactement "un sujet», souvent par opposition à d'autres formes de subjectivité concurrentes. L'unité du sujet se manifeste dans l'espace et dans le temps : l'écriture de l'histoire permet au sujet de se déployer sur le plan idéel depuis les origines. Donc pour qu'il y ait écriture de l'histoire, il faut que la communauté des croyants se soit constituée en un Sujet collectif. Aussi une question décisive demeure, à laquelle ne répondent ni les recherches présentées ni d'ailleurs la littérature musulmane : quand la Communauté musulmane s'est-elle constituée comme un Sujet à part entière? Autre question subsidiaire, mais cruciale : quels sont les moyens qui ont permis de fabriquer ce Sujet collectif ? On ne peut les réduire à la pratique de l'écriture historiographique, qui n'est sans doute que la manifestation du processus, non son moteur.

3 Le second chapitre, qui vient en complément du précédent, traite plus précisément de "l'identité arabe" avant l'islam, en relation avec la question de la langue et de l'écriture arabes. Il concerne l'Arabie préislamique. L'auteur s'appuie dans ce chapitre presque exclusivement sur les travaux de Christian Robin, qui portent la plupart du temps sur l'Arabie du Sud et le Yémen antique. D'où une certaine tendance à avancer des assertions sur le Hijāz en partant de ce cas particulier (selon Ch. R. lui-même), par exemple quand elle discute du vocabulaire de l'organisation sociale en arabe (qabila, ' ashïra, sha'b...). Elle conteste la notion de " tribu » appliquée à l'univers arabe (p. 65-66) et dénie un rôle quelconque à la parenté, en s'appuyant exclusivement sur des données empruntées au cas yéménite: "Les inscriptions sud-arabiques montrent que, dans le Yémen préislamique, le fondement des groupes d'une certaine importance n'est pas un lien de parenté, mais un culte commun, la vénération des mêmes dieux» (p. 64-65). Il est regrettable qu'elle n'ait pas tenu compte de ce que l'on sait depuis longtemps grâce à l'anthroponymie arabe, telle que les sources anciennes la dévoilent. Des noms théophores comme les 'Abd Shams ou 'Abd Dār renvoient également à des dieux tutélaires et d'ailleurs ont été proscrits par l'islam naissant. On trouve également des noms théophores similaires chez les Nabatéens et d'autres groupes sémites (Gabriel, Emmanuel, etc. sont aussi des noms théophores). La référence à un dieu familial ou d'un groupe plus large n'exclut pas que le culte à rendre à ce dieu puisse se fonder sur la parenté.

4 Le troisième chapitre a trait à la biographie du Prophète (le titre du chapitre est emprunté au titre d'un article de J. Chabbi). Là aussi relevons un point faible, majeur : la question de la Sira est déconnectée de celle de la Sunna. Ces deux notions devraient être examinées parallèlement. Il y a quelques années, M. M. Bravmann avait montré qu'à une période primitive, les deux vocables étaient des quasi-synonymes (The Spiritual Background of Early Islam. Studies in Ancient Arab Concepts, Brill, 1972, p. 123-139), mais l'auteur semble ignorer son ouvrage. En effet, il est significatif qu'elle ne s'interroge à aucun moment sur la signification du mot arabe sira avant qu'il acquière la signification technique "Vie du Prophète ». Or en arabe, au départ, sira désigne la «conduite». Aussi on ne peut suivre l'auteur quand elle écrit que le terme signifie "récit de vie » (p.77). C'est le sens que l'historien moderne, soucieux de disposer de sources, privilégie, et qui est presque un contresens. Mais sīra, même à l'époque " classique ", ne distingue pas le « récit de vie » de la dimension édificatrice et même hagiographique.

5 Dans le quatrième chapitre, l'auteur tente de résumer les nombreuses recherches sur le Coran. Elle présente de manière cursive un grand nombre de ces recherches. Elle évoque aussi le cas des manuscrits découverts il y a près d'un demi-siècle à Sanaa au Yémen, dont certains peuvent être très anciens (fin du viI siècle au plus tard) et 
remettent donc sérieusement en cause la thèse d'un Coran d'élaboration tardive, qui avait été défendue hardiment par John Wansbrough. Parmi les autres recherches évoquées, il y a l'hypothèse que le Coran serait issu d'un «lectionnaire» en syriaque. Un «lectionnaire» est un livre dont on se servait pour la liturgie dans les offices syriaques et qui contenait des fragments de la Bible. Cela pourrait expliquer les références fragmentaires à celle-ci dans le Coran. On se serait contenté de les rendre en arabe. Un auteur libanais (et non allemand comme l'écrit l'auteur, p. 119), qui a publié un livre sur le Coran sous un pseudonyme allemand (Christoph Luxenberg), va beaucoup plus loin : il soutient que la langue d'écriture originelle du Coran est le syroaraméen ; c'est pour cela que pour comprendre les passages obscurs, il faut se référer à cet idiome.

6 Le chapitre 5 aborde un problème ancien : comment rendre compte de l'émergence au $\mathrm{VII}^{\mathrm{e}}$ siècle d'un " empire » musulman ? Certains auteurs veulent y voir la conséquence de l'apparition d'une nouvelle religion; d'autres veulent y voir un phénomène purement sociopolitique. Si on ne peut ramener l'expansion de l'islam à quelques proclamations coraniques, il n'en reste pas moins que la religion joua un rôle majeur dans ce phénomène. Un autre aspect du problème est le rapide succès des conquérants.

7 Le chapitre 6 concerne «l'histoire des Califes». Tayeb El-Hibri estime que la version canonique de l'histoire des quatre premiers Califes est une construction purement littéraire, sans fondement historique. Cependant une Chronique syriaque anonyme (775) ne confirme pas cette hypothèse radicale. Des recherches nouvelles insistent sur l'antiCalife 'Abd Allāh b. al-Zubayr, qui défia les Umayyades. L'Auteur attire avec raison l'attention sur la relecture " nationaliste » et par conséquent anachronique de l'histoire de cette période ancienne, notamment de la « révolution » abbasside (132/750). Si la sociologie a pu jouer un rôle, on ne peut non plus en faire la conséquence d'une « lutte des classes »- du reste, dans une telle perspective, il faudrait commencer par définir le mode de production en cause ainsi que ses caractéristiques. L'Égyptien Samir Amin (qui est un économiste et non un historien) avait proposé la notion de « mode de production tributaire", mais qui devait rendre compte de situations très diverses (Égypte ancienne, Mésopotamie, monde islamique, Chine ancienne, Inde ancienne, voire Amérique précolombienne). Le chapitre 7 est étroitement lié au chapitre 6. Selon certains chercheurs, le calife umayyade 'Abd al-Malik b. Marwān a eu un rôle bien plus important que ne l'admet la Tradition musulmane. C'est à son époque qu'aurait eu lieu l'établissement de la vulgate coranique et que la formule « Au nom de Dieu, Muhammad apôtre de Dieu » se répandit. Sur des pièces de monnaie (691-697), apparaît la formule de la shahāda (profession de foi musulmane). Sur des pièces d'argent apparaissent même des citations coraniques, notamment la sourate 112 qui est d'orientation antichrétienne. Un des résultats de diverses recherches est que le nom de Muhammad n'apparaît dans la papyrologie, l'épigraphie ou la numismatique que vers la fin du $\mathrm{VII}^{\mathrm{e}}$ siècle. Si on exclut que ce soit un hasard ou un effet de l'état d'avancement de la recherche, comment rendre compte de ce fait? Plusieurs hypothèses ont été avancées. L'auteur expose les différentes thèses. Certains chercheurs (Wansbrough, Crone, Cook) ont cru en tirer une datation plus tardive de la naissance de l'islam. D'autres (Donner, Hoyland), avec raison, ont proposé d'y voir une métamorphose d'une religion primitive, qui demeure pour le moment difficile à cerner.

8 On peut recommander cet ouvrage aux islamisants débutants comme aux nonislamisants. Toutefois, on doit indiquer qu'un grand nombre de recherches ne sont pas 
évoquées. En raison d'une conception «positiviste » de l'histoire, l'auteur ne souffle aucun mot de l'histoire de la pensée juridico-religieuse. À aucun moment, il n'est question ni de théologie ( $k a l a ̄ m)$, ni de sciences légales (fiqh), ni de soufisme. Il n'est pas non plus question d'histoire de la grammaire, qui est pourtant un domaine majeur. Ce qui est plus surprenant, c'est l'absence totale de toute référence au débat sur le hadith. Nous avons relevé à ce sujet qu'après avoir attribué à tort la méthode du commonlink à Harald Motzki (p. 78), à la page suivante, elle l'attribue à Gauthier Juynboll (p. 79, note 10) ; or, elle est le fait de Joseph Schacht dès 1950 ! Il est vrai que Juynboll l'a reprise et a cherché à la systématiser dans une série d'écrits. 